\section{Uncoupling diffusion and binding in FRAP experiments}

To the Editor: In their recent article, Schwarzenbacher et al. ${ }^{1}$ use an elegant micropatterning technique to demonstrate the effect of immobile CD4 receptors on the mobility of the interacting protein Lck. One powerful feature of protein immobilization methods is the ability to extract binding and unbinding parameters using fluorescence recovery after photobleaching (FRAP) ${ }^{2,3}$. However, in their calculation of CD4-Lck unbinding rates, the authors made a simplifying assumption that introduces a serious risk of error.

Binding of fluorescent molecules to immobile sites will slow recovery after photobleaching provided the binding events are sufficiently frequent and long-lived. The effect of binding on FRAP depends on the relative rates of binding and diffusion. At the extreme where diffusion is much more rapid than binding, fluorescence recovery reflects only unbinding. These circumstances are referred to as diffusion-uncoupled, to distinguish them from instances in which fluorescence recovery is a complex function of both binding and diffusion (diffusion-coupled) ${ }^{3,4}$.

Schwarzenbacher et al. ${ }^{1}$ derive the lifetime of the CD4-Lck interaction after concluding that Lck contrast recovery is diffusion-uncoupled. They made this conclusion after comparing the time required for an Lck molecule to diffuse across a bleached region (the characteristic diffusion time) and the time required for recovery of fluorescence contrast. The diffusion time for full-length $\mathrm{Lck}$ (with a diffusion coefficient of $\sim 0.2-1.0 \mu \mathrm{m}^{2} \mathrm{~s}^{-1}$ ) across $20 \times 5 \mu \mathrm{m}$ rectangles will be $\sim 6-30 \mathrm{~s}$. The authors argue that this is sufficiently rapid compared to the $160 \mathrm{~s}$ required for recovery after photobleaching that diffusion is negligible and thus that the recovery rate corresponds to the unbinding rate. Although this makes intuitive sense, the diffusion-coupled and diffusion-uncoupled regimes cannot be distinguished simply by comparing diffusion and recovery rates ${ }^{4}$. A necessary preliminary step is to determine empirically whether diffusion has an impact on recovery. This is most commonly done by measuring recovery as a function of bleached region size.

Notably, diffusion can be important even for cases in which recovery after photobleaching is extremely slow ${ }^{3,4}$. Estimates have also suggested that diffusion-coupled recovery will be more common than diffusion-uncoupled recovery for most binding partners ${ }^{3}$. Moreover, inappropriately ignoring diffusion can have serious consequences, as derived dissociation rates may be in error by as much as two orders of magnitude ${ }^{5}$. Schwarzenbacher et al. ${ }^{1}$ calculated unbinding rates for full-length (membraneassociated) and truncated (cytosolic) Lck that differed by this amount, and concluded that the Lck membrane anchor has a profound influence on the stability of the protein-protein interaction. The micropatterning method described by the authors represents a valuable addition to the inventory of live-cell protein immobilization techniques. However, as is the case for other photobleaching techniques, extraction of accurate binding and unbinding parameters using this method will require experimental assessment of the impact of diffusion on fluorescence or contrast recovery.

\section{Nevin A Lambert}

Department of Pharmacology and Toxicology, Medical College of Georgia, 1120 15th St., Augusta, Georgia 30912, USA.

e-mail: nlambert@mcg.edu

1. Schwarzenbacher, M. et al. Nat. Methods 5, 1053-1060 (2008).

2. Phair, R.D., Gorski, S.A. \& Misteli, T. Methods Enzymol. 375, 393-414 (2004).

3. Sprague, B.L., Pego, R.L., Stavreva D.A. \& McNally, J.G. Biophys. J. 86, 3473-3495 (2004).

4. Sprague, B.L. \& McNally, J.G. Trends Cell Biol. 15, 84-91 (2005).

5. Sprague, B.L. et al. Biophys. J. 91, 1169-1191 (2006).

Brameshuber et al. reply: We thank Nevin Lambert for bringing this topic to discussion and pointing out the potential pitfalls in fluorescence recovery after photobleaching (FRAP) analysis ${ }^{1}$, which must also be considered in the case of our combined micropattern-FRAP method for the determination of interaction lifetimes ${ }^{2}$. As he pointed out, problems may arise when analyzing the binding of a mobile molecule to an immobile receptor $^{3-5}$. Two scenarios for FRAP in the presence of binding may be distinguished: (i) diffusion-uncoupled FRAP, in which diffusion hardly affects the recovery dynamics (the recovery time, $\tau$, equals the inverse of the off rate); and (ii) diffusion-coupled FRAP, in which probe molecules diffuse into the bleached spot and bind to the first free binding sites they encounter. Recovery proceeds as a succession of binding-unbinding and diffusion steps, and the resulting FRAP curve contains contributions from both diffusion and binding. Here we argue that, for our experimental conditions, the simplified assumption of diffusion-uncoupled FRAP is appropriate.

We provided one argument supporting this assumption in our original manuscript ${ }^{2}$. At the onset of the recovery process, we analyzed the mobility of the first Lck-CFP-YFP molecules entering the bait-captured spot in the photobleached area (Fig. 6 in ref. 2). We reasoned that, in the case of diffusion-coupled FRAP, the probe would move via a succession of short diffusive periods interrupted by transient binding to the bait; in the extreme case of very high on and off rates compared to the single-molecule observation time, a reduced effective diffusion constant would be obtained. However, we found no difference between Lck-CFPYFP molecules moving in the bait-captured versus bait-free area. 

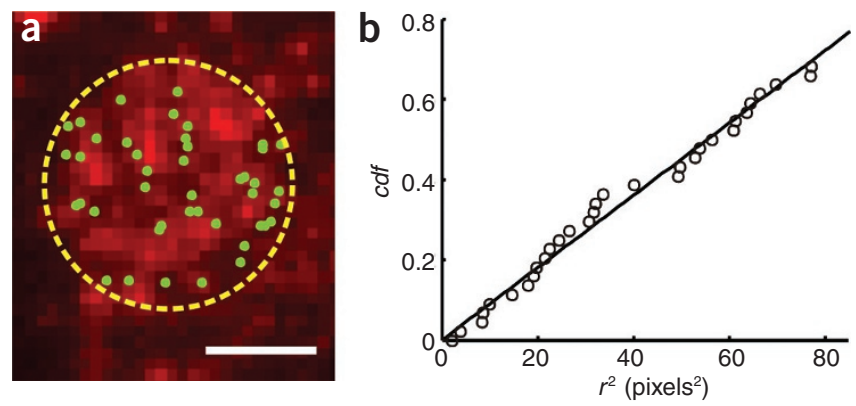

Figure 1 | Spatial profile of the first Lck-CFP-YFP molecules entering the bleached spot. (a) The positions of the first Lck-CFP-YFP molecules entering the bleached spot (dashed circle) are shown as green dots, overlaid onto the rescaled prebleach image (red). We reanalyzed here the bottom spot displayed in Figure 6 of reference 2. Data obtained from multiple FRAP runs on the same spot were pooled. Scale bar, $2 \mu \mathrm{m}$. (b) Distance distribution of Lck-CFP-YFP molecules from the spot center.

In particular, we observed no additional immobilization events in the bait-captured area, indicating that binding to unoccupied CD4 molecules is a very rare event, at least on the millisecond time scale within which the membrane-anchored Lck-CFP-YFP molecules moved over the photobleached spot.

In addition, here we provide additional analysis of the spatial distribution of these Lck-CFP-YFP molecules within the bleached bait-captured spot (Fig. 1a). For diffusion-coupled FRAP, the succession of binding-unbinding events of the probe during its recovery would yield a gradual increase of the fluorescence toward the boundary of the spot. In contrast, Lck-CFPYFP was distributed homogenously over the entire spot area. We calculated the distance of each molecule from the spot center, $\rho$, and plotted the cumulative density function cdf $(r)=\mathrm{P}(\rho \leq r)$ denoting the probability for $\rho$ to be smaller or equal to the value $r$ (Fig. 1b). The curve follows a linear relationship according to $\operatorname{cdf}\left(r^{2}\right)=(r / R)^{2}$, with $\mathrm{R}$ denoting the spot radius, confirming the uniform probe distribution. Thus, the observed Lck-CFP-YFP diffusion and distribution within the spot justify the use of a diffusion-uncoupled FRAP model.

\section{ACKNOWLEDGMENTS}

This work was supported by the Austrian Science Fund (project Y250-B10), the Competence Center for Biomolecular Therapeutics Research-Vienna and by the GEN-AU project of the Austrian Federal Ministry for Science and Research.

Mario Brameshuber ${ }^{1}$, Michaela Schwarzenbacher ${ }^{1}$, Martin Kaltenbrunner ${ }^{1}$, Clemens Hesch ${ }^{1}$, Wolfgang Paster ${ }^{2}$, Julian Weghuber ${ }^{1}$, Bettina Heise ${ }^{3}$, Alois Sonnleitner ${ }^{4}$, Hannes Stockinger ${ }^{2} \&$ Gerhard J Schütz ${ }^{1}$

${ }^{1}$ Biophysics Institute, Johannes Kepler University Linz, Altenbergerstr. 69, A-4040 Linz, Austria. ${ }^{2}$ Department of Molecular Immunology, Center for Physiology, Pathophysiology and Immunology, Medical University of Vienna, Lazarettgasse 19, A-1090 Vienna, Austria. ${ }^{3}$ Department of Knowledge-based Mathematical Systems, Johannes Kepler University Linz, Altenbergerstr. 69, A-4040 Linz, Austria. ${ }^{4}$ Center for Biomedical Nanotechnology, Upper Austrian Research GmbH, Scharitzerstr. 6-8, A-4020 Linz, Austria.

e-mail: gerhard.schuetz@jku.at or hannes.stockinger@meduniwien.ac.at

1. Lambert, N.A. Nat. Methods 6, 183 (2009).

2. Schwarzenbacher, M. et al. Nat. Methods 5, 1053-1060 (2008).

3. Spraque, B.L. \& McNally, J.G. Trends Cell Biol. 15, 84-91 (2005).

4. Sprague, B.L. et al. Biophys. J. 91, 1169-1191 (2006).

5. Sprague, B.L., Pego, R.L., Stavreva, D.A. \& McNally, J.G. Biophys. J. 86, 34733495 (2004).

\section{A computational approach to correct arginine-to-proline conversion in quantitative proteomics}

To the Editor: Stable-isotope labeling of proteins using heavy amino acids in cell culture is a widely used method to measure quantitative changes in mass spectrometry-based proteomics ${ }^{1}$. When using a heavy form of arginine, however, 'heavy'-isotope labels can be inserted into proline through arginine catabolism. If the stable isotope incorporated into proline is not considered, ratios of proline-containing light and heavy peptides can be incorrectly calculated, leading to a reduction in intensity of the isotope-labeled heavy peptide (Fig. 1a). Proposed solutions have included decreasing the arginine concentration $^{2}$ or increasing the proline concentration ${ }^{3}$, but manipulating the amino acid concentration in culture medium may result in suboptimal growth conditions for certain cell lines ${ }^{4}$. Another method replaces ${ }^{12} \mathrm{C}^{14} \mathrm{~N}$ arginine with ${ }^{12} \mathrm{C}^{15} \mathrm{~N}$ arginine in the 'light' medium, allowing the amount of converted proline to be normalized by quantifying the monoisotopic peak in the mass spectra ${ }^{4}$. However, quantification solely based on monoisotopic peak can also compromise accuracy.

We describe an alternative, computational correction for accurate calculation of expression ratios between 'light' and 'heavy' isotopelabeled peptides, which does not require changing the culture medium used for labeling. In a high-resolution mass spectrometer, isotope clusters are well defined, making it straightforward to distinguish the converted heavy proline clusters from the heavy arginine clusters (Fig. 1a). To correct for proline conversion, we extracted individual isotope peaks rather than simply summing all ion intensities within a massto-charge $(\mathrm{m} / \mathrm{z})$ range of a predicted isotope distribution in which noisy peaks can be potentially included. We summed ion current from multiple light isotope peaks with a defined mass window to calculate the amount of light peptide. For the heavy peptide, we summed ion current not only from the heavy arginine- or heavy lysine-labeled peptide counterpart of the lighter peptide but also from extra heavy isotope peaks derived from heavy proline (Fig. 1b).

Using an LTQ Orbitrap mass spectrometer (Thermo Fisher Scientific), we analyzed a 1:1 mixture of cultured cortical neurons grown in light or heavy media. Owing to the nature of the primary cell culture (cells were not doubling) used, we observed incomplete heavy-isotope labeling (60-90\% $)^{5}$. By using the ratio distribution of no proline-containing peptides (Fig. 1c) as the target for the correction, we plotted the distribution with a single-proline correction; the distribution had a shift toward the expected ratio and becomes more focused, indicating a more accurate overall ratio calculation. When considering the second heavy proline, the distribution plot was almost identical to the expected ratio. For PC12 cells that we completely labeled with heavy isotope, the proline correction resulted in a complete overlap of the ratio distribution profile between the proline-containing and no proline-containing peptides, and the distribution was centered at zero (Fig. 1d). We also applied this approach to the same data in a low-resolution mode (we simply summed all ion intensities within the predicted isotope distribution's $m / z$ range with $0.3 \mathrm{~m} / z$ tolerance on each side) where both single and double proline correction improved the accuracy of the ratio (Supplementary Methods, Supplementary Fig. 1 and Supplementary Table 1 online).

Depending on cell type and media conditions, heavy proline can contribute up to $30-40 \%$ of the proline-containing peptide abun- 